\title{
Prevalence of and risk factors for increased serum levels of allergen-specific $\lg E$ in a population of Norwegian dogs
}

\author{
Annelin A Bjelland ${ }^{1 *}$, Frederik L Dolva ${ }^{1}$, Ane Nødtvedt ${ }^{2}$ and Bente K Sævik ${ }^{3}$
}

\begin{abstract}
Background: The importance of different allergens in association with IgE production and canine atopic dermatitis (CAD) has been poorly studied and few studies exist on factors influencing allergen-specific IgE antibodies in serum. The aim of this cross-sectional study was to investigate the prevalence of elevated lgE levels to different environmental allergens in Norwegian dogs with a suspicion of CAD. The secondary aim was to identify risk factors associated with elevated serum levels of allergen-specific lgE.

Results: The study sample consisted of serum from 1313 dogs of 161 different breeds. All samples were submitted for serologic IgE-testing (Fc epsilon R1 alpha-based ELISA) based on suspicion of CAD. Overall, 84.3\% of the dogs had elevated IgE levels to one or more of the allergen(s). The predominant allergens amongst the positive results were the indoor allergens (Acarus siro 84.0\%, Dermatophagoides farinae 80.2\%, Tyrophagus putrescentiae 79.9\%). Sheep sorrel was the most commonly encountered outdoor allergen (40.0\%). Only $2.6 \%$ of the dogs with elevated IgE levels were positive to flea saliva.

The test results varied significantly depending on when the serum samples were taken. Samples taken during summer and autumn more often came out positive than samples taken during winter and spring.

Geographical variations were also demonstrated. A greater proportion of females than males had positive test results, and more females than males tested positive to outdoor allergens. The mean age was significantly higher in the dogs testing positive than amongst the dogs testing negative. The allergen-specific IgE levels varied with breed. The boxer was the only breed with a significantly higher proportion of positive test results compared to the other breeds. Boxers also had a higher prevalence of elevated lgE levels to outdoor allergens, whereas the Rottweiler had a higher prevalence of elevated lgE levels to indoor allergens compared to the other breeds.

Conclusions: IgE hypersensitivity was most often associated with indoor allergens. Outdoor allergens were of minor importance and lgE reactivity to flea saliva was rare. Breed differences in allergen-specific lgE levels were identified. Season of sampling, and the dogs' geographical localisation, sex and age also affected the results of the lgE analysis.
\end{abstract}

Keywords: Canine, Allergens, Immunoglobulins, Epidemiology, Serology, Allergy testing

\section{Background}

Canine atopic dermatitis (CAD) is a commonly encountered disease in small animal practice. It is defined as "a genetically predisposed inflammatory and pruritic allergic skin disease with characteristic clinical features, most commonly associated with IgE antibodies to environmental

\footnotetext{
* Correspondence: bjellandannelin@gmail.com

'Department of Companion Animal Clinical Sciences, Faculty of Veterinary Medicine and Biosciences, Norwegian University of Life Sciences, PO box 8146 Dep., NO-0033, Oslo, Norway

Full list of author information is available at the end of the article
}

allergens" [1]. Epidermal antigens, mite antigens, house dust, insect antigens, pollens and mould spores are examples of allergen groups associated with CAD [2].

Interaction between allergen-specific IgE antibodies and the relevant allergens is widely accepted as an important part of the pathogenesis of CAD, although it is known that CAD is a multifactorial disease that cannot be explained by IgE-mediated hypersensitivity alone $[3,4]$. Cutaneous antigen-presenting cells (Langerhans cells),

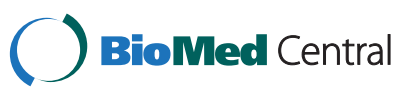


$\mathrm{T}$ lymphocytes as well as many other cells and inflammatory mediators are all contributing to the disease process [4]. Also, a defective epidermal barrier function is thought to allow for the penetration of environmental allergens [4-6]. Many authors have investigated risk factors for the development of CAD. Geographical variations in the prevalence of the disease indicate that a dog's risk of developing CAD is influenced by geographical localisation. Climate, vegetation, life style, population density and pollution levels are all factors that might contribute to the varying occurrence of CAD in different regions [7-9]. Several studies document that certain breeds are overrepresented with the disease, indicating a genetic predisposition. However, the reported breeds vary between different publications. This could be explained by geographical and temporal differences in the genetic material of dog breeds, but might also be a result of different study populations $[7,10,11]$. There is no known sex predilection to CAD [12].

The initial diagnosis of CAD should be based on a set of clinical criteria; the currently favoured set was proposed by Favrot et al. [13]. It is also essential to exclude differential diagnoses, as well as carrying out an elimination diet trial to document whether food allergens are involved in the development of the disease [12,13]. After performing a proper work-up leading to the clinical diagnosis of CAD, IgE-based serologic or intradermal testing can be used to investigate whether or not the clinical signs are associated with allergen-specific IgE. Negative results to these tests occur in patients with atopiclike dermatitis (ALD), which is a disease with the same clinical features as CAD but with no detectable IgE response to allergens. Further, serologic and intradermal testing is useful to identify which allergens to tentatively reduce or eliminate from the patient's environment or which allergens to include in immunotherapy [12].

The influence of potential risk factors on serum levels of allergen-specific IgE has been poorly studied in the dog. It seems likely that geographical factors have an impact on the results of serologic IgE tests, as dogs in different regions are exposed to varying amounts of different allergens. Also, seasonal differences in allergen exposure presumably affect the serum levels of allergen-specific IgE [14]. Whether or not birth season influence allergenspecific IgE production has not been investigated, and studies on the association between birth season and CAD have shown conflicting results $[7,15,16]$. Breed differences in serum IgE levels have been documented in a few studies, indicating that the production of IgE antibodies is influenced by genetic factors $[17,18]$. There are conflicting results regarding the impact of a dog's age and sex on serum levels of IgE [17-19]. Vaccination regimens, parasitic disease and glucocorticoid administration are other factors that can influence serum IgE levels in dogs [14].
The prevalence of elevated IgE levels to specific allergens has been poorly studied in Norwegian dogs. Worldwide, only a few studies exist on factors that may increase the risk of developing elevated allergen-specific IgE levels in canine serum. Hence, the aim of the present study was to investigate the prevalence of elevated serum levels of IgE to different environmental allergens in serum samples from Norwegian dogs that underwent serologic allergen testing. A secondary aim was to identify risk factors associated with elevated serum levels of allergen-specific IgE.

\section{Materials and methods Study sample}

In this cross-sectional study, the material was obtained from Dr. Baddaky AS (Skotterud, Norway), which is the only Norwegian enterprise to analyze allergen-specific IgE in animals. The full dataset consisted of serum samples from 1313 dogs, submitted to Dr. Baddaky AS for measurement of allergen-specific IgE (Heska Allercept ${ }^{\circ}$ Serum IgE Test Nordic Panel) between March 2009 and September 2012. The serum samples were taken by veterinarians working in various geographic regions of Norway and were presumably submitted for IgE analysis based on suspicion of CAD.

Forms containing information from the referring veterinarians followed the serum samples and were registered in Dr. Baddaky's database. Information regarding when the samples were taken (the sample date), the postal codes of the submitting veterinarians, each dog's breed, sex and date of birth as well as the results from the IgE analysis were obtained from the database.

\section{IgE-testing}

Heska Allercept ${ }^{\oplus}$ Serum IgE Test is an ELISA-based IgE analysis using the alpha chain of the human high affinity IgE receptor FceRI as the IgE-specific reactant. The Nordic Panel includes indoor and outdoor allergens commonly occurring in Norway. The indoor allergens included in the Nordic Panel are the flour mite Acarus siro, the storage mite Tyrophagus putrescentiae and the house dust mite Dermatophagoides farinae. The outdoor allergens included are timothy grass (Phleum pratense), cocksfoot (Dactylis glomerata), blue grass annual (Poa annua), perennial rye grass (Lolium perenne), sheep sorrel (Rumex sp.), english plantain (Plantago lanceolatum), nettle (Urtica dioica), lambs quarter (Chenopodium sp.), mugwort (Artemisia vulgaris), alder (Alnus sp.), oak (Quercus sp.), birch (Betula sp.), meadow fescue (Festuca elatior), velvet grass (Holcos lanatus), redtop grass (Agrostis sp.), elm (Ulmus sp.), beech (Fagus sylvatica), hazel (Corylus sp.), sycamore (Acer pseudoplatanus) and ragweed (Ambrosia sp.). Flea saliva allergen is also included in the analysis. 
The test results are given as a number on a continuous scale, ranging from 0 to 3000 (for some allergens 4000) enzyme linked immunosorbent assay (ELISA) absorbance units (EA units). This represents the amount of allergen-specific IgE measured in a serum sample. More information about the test procedure is available in the references [20-22].

A test result of 150 EA units or higher was considered positive. The dogs comprising the study sample were sorted according to their individual test results, providing the distribution of test results in the study sample.

\section{Risk factors \\ Breed}

Based on the international breed standards (Federation Cynologique Internationale, FCI), the dogs were categorized by their respective breed, giving a total of 161 different breeds. All mixed breeds were collectively called mixed breed.

\section{Sex}

Each dog was categorized as either male or female, based on the information from the submitting veterinarians. Neutered individuals were not separated into isolated categories, because of uncertainty regarding the completeness of this information. Routine spay/neutering of healthy dogs is not commonly practiced in Norway.

\section{Age}

Each dog's age in months was calculated by subtracting the birth date from the sample date. The dogs were categorized into ten age categories: 0-6 months, $>6$ months -1 year, $>1-2$ years, $>2-3$ years, $>3-4$ years, $>4-5$ years, $>5-6$ years, $>6-7$ years, $>7-8$ years and $>8$ years.

\section{Season}

Each dog's birth season was defined based on the birth date: Spring (March, April, May), summer (June, July, August), autumn (September, October, November) and winter (December, January, February). Season of sampling was categorized in the same manner, depending on the sample date.

\section{Geographical region}

The postal codes of the submitting veterinarians were used to identify the geographical distribution of the study sample. Counties were generated from the postal codes [23]. The 19 counties of Norway were then sorted into seven regions based on a national standard of regional division [24]: Oslo/ Akershus (Oslo, Akershus), Hedmark/Oppland (Hedmark, Oppland), Southeast (Østfold, Buskerud, Vestfold, Telemark), Agder/Rogaland (Aust-Agder, Vest-Agder, Rogaland), Northwest (Hordaland, Sogn og Fjordane, Møre og Romsdal),
Trøndelag (Sør-Trøndelag, Nord-Trøndelag) and North (Nordland, Troms, Finnmark).

\section{Statistical analysis}

The data was transferred into a database created in Excel $^{\circledR}$ (Microsoft $^{\bullet}$ Excel $^{\bullet}$ for Mac 2011, Version 14.3.8.). The dataset was sorted in pivot tables, and all tables and graphs were created using a filtering function in Excel ${ }^{\circ}$.

The mean age of the dogs in the study sample was estimated with a 95\% confidence interval (CI) using Epitools [25]. Non-overlapping confidence intervals for the mean age in different groups were seen as evidence of a statistically significant age difference.

The relationship between the risk factors sex, birth season, sample season and geographical region and the results from the allergen-specific IgE test (+/-) were tabulated and analyzed using a chi-square test. The chisquare test was performed with a 95\% confidence limit using Epitools [26]. A $P$-value of $<0.05$ was considered statistically significant.

Statistical analysis of breed as a risk factor was performed using the software package Stata SE 12 (Stata statistical software, College Station, Texas, USA). The 95\% confidence limit for the proportion of dogs with positive test results within each breed was calculated. Deviation from the "average breed" (pooled test-result across all breeds) was evaluated based on non-overlapping confidence intervals. The breeds represented with less than 10 individuals in the study sample were excluded from the analysis of breed effect.

\section{Results}

\section{Study sample}

The study sample consisted of serum from 1313 dogs. A total of 161 breeds were represented. Besides mixed breed (123 dogs; 9.4\%), the German shepherd dog was the most numerous breed (102 dogs; 7.8\%). The sex distribution was similar, with 673 males (51.3\%) and 640 females (48.7\%). The age of 106 of the 1313 individuals could not be calculated due to lack of information. The remaining 1207 dogs had a mean age of 3.3 years (39.2 months). As with age, the birth season of 106 dogs could not be defined due to lack of information. Amongst the remaining 1207 dogs, a slight majority were born in the spring (374 dogs; $31.0 \%$ ) and slightly fewer were born in the winter (257 dogs; 21.3\%). 285 dogs (23.6\%) were born in the autumn and 291 dogs (24.1\%) were born in the summer. The season of IgE analysis showed more variation. Most serum samples were taken in the summer (462 of the 1313 samples; 35.2\%) and autumn (400 samples; 30.5\%), while only 181 samples (13.8\%) were taken during the winter months. 270 samples (20.6\%) were taken in the spring. A majority of the 1313 samples were sent from the 
southern parts of Norway, with a total of 1174 (89.4\%) coming from regions south of Trøndelag.

\section{IgE test results}

1107 of the 1313 dogs (84.3\%) had IgE levels of 150 EA units or higher (i.e. positive results) for one or more of the allergen(s) included in the test ( $\mathrm{T}+$ ). 595 dogs (45.3\%) had a positive reaction against at least one outdoor allergen $(\mathrm{O}+)$, while 1018 dogs $(77.5 \%)$ reacted positive against at least one indoor allergen (I+). Most dogs that tested positive had elevated IgE levels for both outdoor and indoor allergens $(\mathrm{O}+$ and $\mathrm{I}+, 506$ of the 1107 dogs, 45.7\%) or indoor allergens alone (O- and $\mathrm{I}+, 46.3 \%)$. Only 89 of the dogs with positive results $(8.0 \%)$ were positive to outdoor allergens alone $(\mathrm{O}+$ and $\mathrm{I}-)$.

The distribution of positive results to each allergen is presented in Table 1. The predominant allergens amongst the positive results were $A$. siro $(930 / 1107 ; 84.0 \%)$, D. farinae $(888 / 1107 ; 80.2 \%)$ and T. putrescentiae (885/1107; 79.9\%). Sheep sorrel was the most commonly encountered outdoor allergen, with 443 of the 1107 dogs (40.0\%) testing positive. Elevated IgE levels to flea saliva was by far the least common result in the study sample, with 29 of the 1107 dogs (2.6\%) testing positive. Only two dogs tested positive to flea saliva alone.

\section{Risk factors \\ Sex}

The association between sex and results of the IgE analysis are shown in Table 2. A greater proportion of females than males had positive test results $(\mathrm{T}+; P=0.035)$, and more females tested positive to outdoor allergens than did males $(\mathrm{O}+; P<0.0001)$. No statistically significant difference between sexes was detected for indoor allergens.

\section{Age}

The age was known for 1022 of the 1107 dogs testing positive $(\mathrm{T}+)$. Only one of the positive individuals $(0.1 \%)$ was younger than six months, while 42 dogs $(4.1 \%)$ were between six and twelve months of age. A majority of the dogs with positive results were between one and four years old (679 dogs; $66.4 \%)$. The age category $>1-2$ years was the most numerous (272 dogs; $26.6 \%$ ). The number of positive dogs then decreased with advancing age.

The mean age was significantly higher in the dogs testing positive ( $\mathrm{T}+$; mean age 39.6 months; $95 \%$ CI: 39.339.9 months) than amongst the dogs with negative results (T-; mean age 37.3 months; 95\% CI: 36.6-38.1). The same significant variation was seen for outdoor allergens $(\mathrm{O}+$; mean age 40.6 months; 95\% CI: 40.2-41.1 and O-; mean age 38.1 months; $95 \%$ CI: 37.7-38.5), while the mean age of the dogs testing positive and negative to indoor allergens, respectively, was not significantly different (I+; mean
Table 1 The number and proportion of elevated serum levels of allergen-specific IgE to each allergen in a database of test results from serologic allergen-specific lgE-testing in Norwegian dogs

\begin{tabular}{|c|c|c|}
\hline Allergen & Number & Proportion (\%) \\
\hline \multicolumn{3}{|l|}{ Outdoor allergens } \\
\hline Sheep sorrel & 443 & 40.0 \\
\hline Redtop grass & 415 & 37.5 \\
\hline Cocksfoot & 413 & 37.3 \\
\hline Meadow fescue & 405 & 36.6 \\
\hline Beech & 401 & 36.2 \\
\hline Velvet grass & 388 & 35.0 \\
\hline Perennial rye grass & 385 & 34.8 \\
\hline Timothy grass & 368 & 33.2 \\
\hline Blue grass anual & 334 & 30.2 \\
\hline Ragweed & 288 & 26.0 \\
\hline English plantain & 278 & 25.1 \\
\hline Elm & 275 & 24.8 \\
\hline Lambs quarter & 261 & 23.6 \\
\hline Hazel & 244 & 22.0 \\
\hline Alder & 232 & 21.0 \\
\hline Oak & 226 & 20.4 \\
\hline Sycamore & 219 & 19.8 \\
\hline Mugwort & 219 & 19.8 \\
\hline Birch & 183 & 16.5 \\
\hline Nettle & 136 & 12.3 \\
\hline \multicolumn{3}{|l|}{ Indoor allergens } \\
\hline Acarus siro & 930 & 84.0 \\
\hline Dermatophagoides farinae & 888 & 80.2 \\
\hline Tyrophagus putrescentiae & 885 & 79.9 \\
\hline Flea saliva & 29 & 2.6 \\
\hline
\end{tabular}

Number of samples with $\lg E>150$ EA units, and the corresponding proportion (\%) of all samples with positive results.

age 37.7 months; 95\% CI: 37.1-38.3 and $I-$; mean age 38.1 months; 37.7-38.5).

\section{Birth season}

No statistically significant association could be found between the dog's birth season and results of the IgE analysis. Amongst the dogs born during spring, 318 $(85.0 \%)$ had positive test results $(\mathrm{T}+)$, while 56 dogs (15.0\%) were negative to all allergens (T-). 253 of the dogs born in the summer (86.9\%), 239 of the dogs born in the autumn (83.9\%) and 212 of the dogs born in the winter $(82.5 \%)$ had positive test results $(\mathrm{T}+)$. The corresponding $P$-value, as calculated by chi-squared test, was 0.5166. The relationship between birth season and 
Table 2 The distribution of test results in males and females in a database of test results from serologic allergen-specific IgE-testing in Norwegian dogs

\begin{tabular}{llll}
\hline \multicolumn{1}{l}{ Test result } & \multicolumn{1}{c}{$\begin{array}{l}\text { Male } \\
\text { Number (\%) }\end{array}$} & $\begin{array}{l}\text { Female } \\
\text { Number (\%) }\end{array}$ & $P$-value \\
\hline Overall (any allergen) & & \\
T+ & $553(82.2)$ & $554(86.6)$ & 0.0347 \\
T- & $120(17.8)$ & $86(13.4)$ & \\
Outdoor allergens & & & \\
O+ & $269(40.0)$ & $326(50.9)$ & $<0.0001$ \\
O- & $404(60.0)$ & $314(49.1)$ & \\
Indoor allergens & & & \\
I+ & $508(75.5)$ & $510(79.7)$ & 0.0787 \\
I- & $165(24.5)$ & $130(20.3)$ & \\
\hline A P-value of
\end{tabular}

A $P$-value of $<0.05$ implies a statistically significant difference between the sexes based on a chi-square test.

results to outdoor and indoor allergens $(\mathrm{O}+/ \mathrm{O}-$ and $\mathrm{I}+/ \mathrm{I}-)$ also was insignificant ( $P$-value 0.7124 and 0.3303 , respectively).

\section{Sample season}

The test results varied significantly depending on when the serum samples were taken (Table 3). Samples taken during summer and autumn more often came out positive $(\mathrm{T}+)$ than samples taken during winter and spring. The same pattern was identified in terms of serum IgE levels to outdoor and indoor allergens, respectively.

\section{Geographical region}

The relationship between the dog's geographical region and results of the IgE analysis were statistically significant. The region North had the lowest proportion of positive results $(\mathrm{T}+; 76 \%$ of all samples coming from this region), while the southern regions Agder/Rogaland and Southeast had the highest proportion of positive results
(90.4\% and $91.1 \%$, respectively). The proportion of positive test results amongst the remaining regions varied between 80.2\% (Northwest) and 84.4\% (Trøndelag). The $P$-value for the geographical distribution of positive and negative test results $(\mathrm{T}+/ \mathrm{T}-)$, as calculated by chi-squared test, was 0.0008 .

Oslo/Akershus had the highest proportion of dogs testing positive to outdoor allergens $(\mathrm{O}+; 52.8 \%$ of all samples coming from this region), with the southern regions Agder/ Rogaland and Southeast having the second highest proportion of positive results to outdoor allergens $(51.1 \%$ and $51.1 \%$, respectively). Northwest had the lowest proportion of positive results to outdoor allergens (32.6\%). The remaining regions had the following distribution of positive results to outdoor allergens $(\mathrm{O}+)$ : North $40.0 \%$, Hedmark/ Oppland 42.6\%, Trøndelag 45.3\%. The P-value for the geographical distribution of positive and negative results to outdoor allergens $(\mathrm{O}+/ \mathrm{O}-)$, was $<0.0001$.

The same $P$-value $(<0.0001)$ was calculated for the geographical distribution of positive and negative results to indoor allergens (I+/I-). Agder/Rogaland had the highest proportion of dogs testing positive to indoor allergens (I+; $87.5 \%$ of all samples coming from this region). The region North had the lowest proportion of positive results to indoor allergens $(60.0 \%)$. The proportion of positive results to indoor allergens amongst the remaining regions varied between $74.1 \%$ (Oslo/ Akershus) and 83.7\% (Southeast).

\section{Breed}

Out of the 161 breeds registered in the study sample, 30 breeds were represented with at least 10 individuals. A total of 915 dogs were represented amongst these 30 breeds. The distribution of positive test results within these breeds is shown in the Tables 4, 5 and 6. The boxer was the only breed with a significantly higher proportion of positive test results compared to the overall

Table 3 The distribution of test results in samples taken during different seasons of the year in a database of test results from serologic allergen-specific IgE-testing in Norwegian dogs

\begin{tabular}{lllll}
\hline Test result & $\begin{array}{l}\text { Spring } \\
\text { Number (\%) }\end{array}$ & $\begin{array}{l}\text { Summer } \\
\text { Number (\%) }\end{array}$ & $\begin{array}{l}\text { Autumn } \\
\text { Number (\%) }\end{array}$ & $\begin{array}{l}\text { Winter } \\
\text { Number (\%) }\end{array}$ \\
\hline $\begin{array}{l}\text { Overall (any allergen) } \\
\text { T+ }\end{array}$ & $214(79.3)$ & $399(86.4)$ & $350(87.5)$ & $144(79.6)$ \\
T- & $56(20.7)$ & $63(13.6)$ & $50(12.5)$ & $37(20.4)$ \\
Outdoor allergens & & & & 0.005 \\
O+ & $106(39.3)$ & $220(47.6)$ & $199(49.8)$ & $70(38.7)$ \\
O- & $164(60.7)$ & $242(52.4)$ & $201(50.3)$ & $111(61.3)$ \\
Indoor allergens & $188(69.6)$ & $373(80.7)$ & $319(79.8)$ & $138(76.2)$ \\
I+ & $82(30.4)$ & $89(19.3)$ & $81(20.2)$ & $43(23.8)$ \\
\hline-
\end{tabular}

A $P$-value of $<0.05$ implies a statistically significant difference among the different seasons based on a chi-square test. 
Table 4 The breed distribution of elevated serum IgE levels to all allergens

\begin{tabular}{|c|c|c|c|c|}
\hline Breed & $\begin{array}{l}\text { Number of results in } \\
\text { the study sample }\end{array}$ & Number T+ & Proportion T+ & $\begin{array}{l}95 \% \mathrm{Cl} \text { for } \\
\text { proportion } \mathrm{T}+\end{array}$ \\
\hline Total (all breeds) & 915 & 789 & 0.86 & $0.84-0.89$ \\
\hline American cocker spaniel & 17 & 15 & 0.88 & $0.64-0.99$ \\
\hline American Staffordshire terrier & 10 & 8 & 0.8 & $0.44-0.97$ \\
\hline Bernese mountain dog & 11 & 10 & 0.91 & $0.59-1.00$ \\
\hline Bichon frisé & 26 & 22 & 0.85 & $0.65-0.96$ \\
\hline Mixed breed & 123 & 112 & 0.91 & $0.85-0.95$ \\
\hline Dogue de Bordeaux & 10 & 7 & 0.7 & $0.35-0.93$ \\
\hline Boxer & 59 & 58 & 0.98 & $0.91-1.00$ \\
\hline Bull terrier & 13 & 10 & 0.77 & $0.46-0.95$ \\
\hline Cavalier King Charles spaniel & 15 & 10 & 0.67 & $0.38-0.88$ \\
\hline Chinese crested dog & 10 & 9 & 0.9 & $0.55-1.00$ \\
\hline English cocker spaniel & 16 & 12 & 0.75 & $0.48-0.93$ \\
\hline Dachshond short hair & 10 & 10 & 1 & $0.69-1.00^{*}$ \\
\hline Dachshund wire-hair & 10 & 10 & 1 & $0.69-1.00^{*}$ \\
\hline Dalmatian & 12 & 11 & 0.92 & $0.62-1.00$ \\
\hline Bulldog & 16 & 13 & 0.81 & $0.54-0.96$ \\
\hline English setter & 38 & 27 & 0.71 & $0.54-0.85$ \\
\hline Flat-coated retriever & 24 & 19 & 0.79 & $0.59-0.93$ \\
\hline French bulldog & 25 & 22 & 0.88 & $0.69-0.97$ \\
\hline Golden retriever & 56 & 51 & 0.91 & $0.80-0.97$ \\
\hline Gordon setter & 23 & 22 & 0.96 & $0.78-1.00$ \\
\hline Soft-coated wheaten terrier & 16 & 13 & 0.81 & $0.54-0.96$ \\
\hline Jack Russell terrier & 29 & 19 & 0.66 & $0.47-0.82$ \\
\hline Labrador retriever & 83 & 74 & 0.89 & $0.80-0.95$ \\
\hline Pug & 17 & 10 & 0.59 & $0.33-0.82$ \\
\hline Newfoundland & 14 & 13 & 0.93 & $0.66-1.00$ \\
\hline Nova Scotia duck tolling retriever & 12 & 12 & 1 & $0.74-1.00^{*}$ \\
\hline Rottweiler & 43 & 42 & 0.98 & $0.88-1.00$ \\
\hline German shepherd dog & 102 & 82 & 0.8 & $0.71-0.86$ \\
\hline Staffordshire bull terrier & 61 & 53 & 0.87 & $0.76-0.94$ \\
\hline Tibetan spaniel & 14 & 13 & 0.93 & $0.66-0.88$ \\
\hline
\end{tabular}

*One sided $97.5 \% \mathrm{Cl}$.

Number of samples, number of test positive samples $(T+)$, proportion of $T+$ and $95 \%$ confidence interval $(\mathrm{Cl})$ for the proportion of $T+$ of all samples from the relevant breed.

average across breeds $(\mathrm{T}+; 58$ out of the 59 boxers; 98\%). Pugs and Jack Russell terriers, on the other hand, had a significantly lower proportion of positive test results $(\mathrm{T}+; 59 \%$ and $66 \%$, respectively). The boxer also was the breed with the highest proportion of positive results to outdoor allergens $(\mathrm{O}+; 73 \%)$, while the flat-coated retriever and the German shepherd dog had the lowest proportion of dogs testing positive to these allergens $(\mathrm{O}+$; $17 \%$ and $29 \%$, respectively). The highest proportion of positive results to indoor allergens was found in the Rottweiler (I+; 98\%), while the English setter had the lowest proportion of positive results to these allergens (I+; 55\%).

\section{Discussion}

Prevalence of elevated serum IgE levels to environmental allergens

A large majority $(84.3 \%)$ of the dogs in the present study had positive results to one or more of the allergens included in the Nordic panel serum IgE test. The actual prevalence of elevated IgE levels in the general dog population is not known [9], although it is reasonable to believe that the prevalence is lower than the results from this study. The high proportion of positive results might be caused by a combination of factors. Firstly, the serum samples were presumably submitted for IgE analysis 
Table 5 The breed distribution of elevated serum IgE levels to outdoor allergens

\begin{tabular}{|c|c|c|c|c|}
\hline Breed & $\begin{array}{l}\text { Number of results } \\
\text { in study sample }\end{array}$ & Number $\mathrm{O+}$ & Proportion O+ & $\begin{array}{l}95 \% \mathrm{Cl} \text { for } \\
\text { proportion O+ }\end{array}$ \\
\hline Total (all breeds) & 915 & 422 & 0.46 & $0.43-0.49$ \\
\hline American cocker spaniel & 17 & 8 & 0.47 & $0.23-0.72$ \\
\hline American Staffordshire terrier & 10 & 5 & 0.5 & $0.19-0.81$ \\
\hline Bernese mountain dog & 11 & 3 & 0.27 & $0.06-0.61$ \\
\hline Bichon frisé & 26 & 12 & 0.46 & $0.27-0.67$ \\
\hline Mixed breed & 123 & 69 & 0.56 & $0.47-0.65$ \\
\hline Dogue de Bordeaux & 10 & 4 & 0.4 & $0.12-0.74$ \\
\hline Boxer & 59 & 43 & 0.73 & $0.60-0.84$ \\
\hline Bull terrier & 13 & 3 & 0.23 & $0.05-0.54$ \\
\hline Cavalier King Charles spaniel & 15 & 5 & 0.33 & $0.12-0.62$ \\
\hline Chinese crested Dog & 10 & 5 & 0.5 & $0.19-0.81$ \\
\hline English cocker spaniel & 16 & 4 & 0.25 & $0.07-0.52$ \\
\hline Dachshund short hair & 10 & 5 & 0.5 & $0.19-0.81$ \\
\hline Dachshund wire-hair & 10 & 6 & 0.6 & $0.26-0.88$ \\
\hline Dalmatian & 12 & 8 & 0.67 & $0.35-0.90$ \\
\hline Bulldog & 16 & 4 & 0.25 & $0.07-0.52$ \\
\hline English setter & 38 & 23 & 0.61 & $0.43-0.76$ \\
\hline Flat-coated retriever & 24 & 4 & 0.17 & $0.05-0.37$ \\
\hline French bulldog & 25 & 11 & 0.44 & $0.24-0.65$ \\
\hline Golden retriever & 56 & 28 & 0.5 & $0.36-0.64$ \\
\hline Gordon setter & 23 & 16 & 0.7 & $0.47-0.87$ \\
\hline Soft-coated wheaten terrier & 16 & 8 & 0.5 & $0.25-0.75$ \\
\hline Jack Russell terrier & 29 & 10 & 0.34 & $0.18-0.54$ \\
\hline Labrador retriever & 83 & 28 & 0.34 & $0.24-0.45$ \\
\hline Pug & 17 & 4 & 0.24 & $0.07-0.50$ \\
\hline Newfoundland & 14 & 4 & 0.29 & $0.08-0.58$ \\
\hline Nova Scotia duck tolling retriever & 12 & 8 & 0.67 & $0.35-0.90$ \\
\hline Rottweiler & 43 & 23 & 0.53 & $0.38-0.69$ \\
\hline German shepherd dog & 102 & 30 & 0.29 & $0.21-0.39$ \\
\hline Staffordshire bull terrier & 61 & 35 & 0.57 & $0.44-0.70$ \\
\hline Tibetan spaniel & 14 & 6 & 0.43 & $0.18-0.71$ \\
\hline
\end{tabular}

Number of samples, number of test positive samples to outdoor allergens $(\mathrm{O}+)$, proportion of $\mathrm{O}+$ and $95 \%$ confidence interval $(\mathrm{Cl})$ for the proportion $\mathrm{O}+$ of all samples from the relevant breed.

based on suspicion of CAD. However, the diagnostic work-up performed by the submitting veterinarians was not investigated in this study. Provided that the dogs have been correctly diagnosed with CAD before the IgE analysis, the negative testing individuals could represent the diagnosis of atopic-like dermatitis (ALD). Secondly, Dr. Baddaky AS recommends performing Allercept ${ }^{\circ}$ Screening test to measure total IgE levels to different allergen groups (grass pollen, tree pollen and indoor allergens) before embarking upon the allergen-specific assays. The Nordic Panel is recommended following positive results to both indoor and outdoor allergens on the screening test [27].
Hence, the study sample was not randomly selected but represents a subset of dogs in which veterinarians have suspected hypersensitivity to both indoor and outdoor allergens, and no conclusion can be made about the prevalence of elevated allergen-specific IgE levels in the general Norwegian dog population from this study. However, the results can probably be extrapolated to other populations of dogs subjected to allergy testing in other geographic regions.

The predominant allergens amongst the positive results were the indoor allergens: A. siro (84.0\%), D. farinae (80.2\%) and T. putrescentiae (79.9\%). It should be noted 
Table 6 The breed distribution of elevated serum IgE levels to indoor allergens

\begin{tabular}{|c|c|c|c|c|}
\hline Breed & $\begin{array}{l}\text { Number of results } \\
\text { in study sample }\end{array}$ & Number I+ & Proportion I+ & $\begin{array}{l}95 \% \mathrm{Cl} \text { for } \\
\text { proportion I+ }\end{array}$ \\
\hline Total (all breeds) & 915 & 717 & 0.78 & $0.76-0.81$ \\
\hline American cocker spaniel & 17 & 13 & 0.76 & $0.50-0.93$ \\
\hline American Staffordshire terrier & 10 & 8 & 0.8 & $0.44-0.97$ \\
\hline Bernese mountain dog & 11 & 9 & 0.82 & $0.48-0.98$ \\
\hline Bichon frisé & 26 & 19 & 0.73 & $0.52-0.88$ \\
\hline Mixed breed & 123 & 98 & 0.8 & $0.71-0.86$ \\
\hline Dogue de Bordeaux & 10 & 7 & 0.7 & $0.35-0.93$ \\
\hline Boxer & 59 & 49 & 0.83 & $0.71-0.92$ \\
\hline Bull terrier & 13 & 10 & 0.77 & $0.46-0.95$ \\
\hline Cavalier King Charles spaniel & 15 & 10 & 0.67 & $0.38-0.88$ \\
\hline Chinese crested dog & 10 & 7 & 0.7 & $0.35-0.93$ \\
\hline English cocker spaniel & 16 & 11 & 0.69 & $0.41-0.89$ \\
\hline Dachshund short hair & 10 & 9 & 0.9 & $0.55-1.00$ \\
\hline Dachshund wire-hair & 10 & 10 & 1 & $0.69-1.00^{*}$ \\
\hline Dalmatian & 12 & 10 & 0.83 & $0.52-0.98$ \\
\hline Bulldog & 16 & 12 & 0.75 & $0.48-0.93$ \\
\hline English setter & 38 & 21 & 0.55 & $0.38-0.71$ \\
\hline Flat-coated retriever & 24 & 19 & 0.79 & $0.58-0.93$ \\
\hline French bulldog & 25 & 18 & 0.72 & $0.51-0.88$ \\
\hline Golden retriever & 56 & 49 & 0.88 & $0.76-0.95$ \\
\hline Gordon setter & 23 & 21 & 0.91 & $0.72-0.99$ \\
\hline Soft-coated wheaten terrier & 16 & 11 & 0.69 & $0.41-0.89$ \\
\hline Jack Russell terrier & 29 & 17 & 0.59 & $0.39-0.76$ \\
\hline Labrador retriever & 83 & 73 & 0.88 & $0.79-0.94$ \\
\hline Pug & 17 & 9 & 0.53 & $0.28-0.77$ \\
\hline Newfoundland & 14 & 13 & 0.93 & $0.66-1.00$ \\
\hline Nova Scotia duck tolling retriever & 12 & 8 & 0.67 & $0.35-0.90$ \\
\hline Rottweiler & 43 & 42 & 0.98 & $0.88-1.00$ \\
\hline German shepherd dog & 102 & 77 & 0.75 & $0.66-0.83$ \\
\hline Staffordshire bull terrier & 61 & 46 & 0.75 & $0.63-0.86$ \\
\hline Tibetan spaniel & 14 & 11 & 0.79 & $0.49-0.95$ \\
\hline
\end{tabular}

*One sided $97.5 \% \mathrm{Cl}$.

Number of samples, number of test positive samples to indoor allergens (I+), proportion of I+ and $95 \%$ confidence interval (Cl) for the proportion I+ of all samples from the relevant breed.

that the Nordic Panel only includes a subset of selected indoor allergens, and some of these might be false positive results as former studies have documented cross-reactivity amongst the mites [28]. Most dogs testing positive had elevated IgE levels to both indoor and outdoor allergens (45.7\%) or indoor allergens alone (46.3\%). Only $8.0 \%$ of the dogs with positive results were positive to outdoor allergens alone. This agrees well with other studies from the Nordic countries. In a report based on intradermal testing, $D$. farinae was found to be the most important allergen amongst Norwegian dogs with CAD [29]. In a study on a group of 62 atopic dogs from the Nordic countries
(Denmark, Finland, Norway and Sweden), 93.5\% had positive results to house dust mites (D. farinae and/or D. pteronyssinus). Only $16.1 \%$ were positive to pollen allergens in the same study, and none of the dogs reacted to pollen allergens alone. This study was largely based on intradermal testing [30]. Unfortunately, one cannot directly compare our results with these former studies, as there is only partial correlation between serologic and intradermal test results [14]. Several international studies based on serological IgE tests exist, however, the results differ markedly. The lack of a universal standardization of allergen extracts and test methodologies, as well as 
geographical differences in exposure to different allergens are all factors that could explain the heterogeneous results between the different publications. Nevertheless, one can see a general trend from the existing studies. Dermatophagoides spp. seems to be a major allergen in many parts of the world, including Europe. Pollen allergens on the other hand, appear to be important in the USA, but of less importance in Europe $[2,14]$. The present study implies that Norwegian dogs are more prone to develop hypersensitivity to indoor allergens than pollen allergens, similar to the trend reported in other parts of Europe [2].

Elevated IgE levels to flea saliva was by far the least common result in the study sample (2.6\%). This is not surprising, because the flea burden in Norway is relatively low and dogs in areas with low numbers of fleas have a low prevalence of hypersensitivity to flea allergens [31].

\section{Risk factors}

In this study, the dogs' sex, age, breed and geographical region influenced serum levels of allergen-specific IgE. Sampling season also affected the test results, whereas birth season had no significant influence on the IgE levels.

The results from previous studies are ambiguous regarding whether or not there is a sex predilection to elevated serum levels of allergen-specific IgE in dogs [17-19]. In the present study, female dogs had a significantly higher proportion of positive test results than did males. Furthermore, more female than male dogs tested positive to outdoor allergens, whereas no statistically significant difference between sexes was detected for indoor allergens. In conclusion, our results show that female dogs in Norway have a higher risk of developing elevated IgE levels than male dogs, particularly to outdoor allergens. Assuming that IgE antibodies play an essential role in the pathogenesis of CAD and that the dogs have been correctly diagnosed with CAD before the IgE analysis, the results of this study contradict the general consensus that there is no sex predilection to the disease. However, one must keep in mind that neutered and intact individuals were not separated in the present study.

In the current study, the group of dogs with elevated IgE levels to one or more of the allergens had a higher mean age (39.6 months) than the group of dogs with non-elevated IgE levels (37.3 months). Dogs with positive results to outdoor allergens had a higher mean age (40.6 months) than dogs testing negative to the same group of allergens (38.1 months). The age differences were statistically significant, as documented by nonoverlapping confidence intervals. This implies that the production of allergen-specific IgE, particularly to outdoor allergens, varies with age. On the other hand, no significant variation was found in mean age between dogs testing positive and negative to indoor allergens (37.7 and 38.1 months, respectively). Another interesting finding, however, is that dogs with positive results to outdoor allergens had a higher mean age than dogs testing positive to indoor allergens. This could imply that the production of IgE to outdoor allergens is dependent on allergen exposure over a prolonged period of time, compared to the production of IgE to indoor allergens. One could also speculate that Norwegian dogs are more exposed to indoor allergens than outdoor allergens, making elevated IgE levels to indoor allergens appear earlier. The relationship between dogs' age and serum levels of IgE has been poorly studied, and the existing reports are conflicting. A study on healthy beagles found that total serum IgE levels varied with age [19], whereas some of the same authors in another publication concluded that age did not have any influence [18]. In a study on atopic and non-atopic Labrador- and golden retrievers, no association could be found between age and IgE levels, except that the dogs testing positive to flea saliva were older than the dogs testing negative to the same allergen [17].

The present study documents statistically significant geographical variations in canine serum levels of allergenspecific IgE. Several underlying factors could explain this variation. Climate differences might be a factor involved. The region with the lowest proportion of positive results, North, has a lower level of average annual rainfall and lower humidity compared to the other regions [32]. This leads to less suitable conditions for the mites [33], which were found to be the most important allergens in the present study sample. Also, there is less exposure to pollen allergens in North than in other parts of Norway [34]. Population density is another factor that could play a role; North is more sparsely populated than the other regions. To our knowledge, no previous studies on geographical variation in canine allergen-specific IgE have been performed. However, several studies on CAD and atopic diseases in people support the findings in the present study. Average annual rainfall was positively associated with the incidence rate of CAD in a Swedish study, and increasing human population density has been associated with increased occurrence of atopic diseases in both dogs and people $[8,9,15]$. Pollution levels, lifestyle, vaccination routines and parasite control regimes are other geographically varying factors which could contribute to the geographical differences seen in the present and previous studies $[8,9]$.

Significantly higher proportions of the serum samples taken during summer and autumn were positive on the IgE test than samples taken during winter and spring in the present study. This pattern was found for the test overall $(\mathrm{T}+)$, as well as for the outdoor $(\mathrm{O}+)$ and indoor allergens $(\mathrm{I}+)$. The literature is limited in terms of seasonal variations in allergen-specific IgE levels in dogs, 
but season of the year has been found to influence the results of serum IgE tests in people [14]. One could speculate that these findings are caused by seasonal differences in allergen exposure. In Norway, the highest levels of pollen allergens are seen during the spring and summer months [34]. In humans, the IgE levels rise with pollen exposure, peaking approximately four weeks after the exposure, and then declining [14]. If the same is true for dogs, this delay could explain why the proportion of positive results is higher during summer and autumn than during winter and spring. Further, the relative humidity is higher during the warm season than during winter in Norway. Higher humidity levels leads to more suitable conditions for the house dust mite [35], which could explain the results in terms of indoor allergens.

The dogs' birth season did not affect the results of the IgE analysis in this study. To the authors' knowledge, no other published studies have investigated the association between birth season and allergen-specific IgE levels, and results from studies on CAD are conflicting $[7,15,16]$.

The present study documents breed differences in serum levels of allergen-specific IgE. There was a significantly higher proportion of elevated IgE levels $(\mathrm{T}+)$ amongst the boxers than amongst the other breeds in the study sample, indicating that boxers may be more prone to produce IgE antibodies in response to environmental allergens than other breeds. Pugs and Jack Russell terriers seem to be at a lower risk to develop IgE hypersensitivity, as these breeds had a significantly lower proportion of positive results on the IgE test compared to the other breeds represented. Provided that the history of the dogs in the study sample matches the clinical diagnosis of CAD, one could speculate whether pugs and Jack Russell terriers are overrepresented with ALD compared to other breeds.

Further, the present study shows that the propensity to produce IgE antibodies to different allergen groups varies with breed. On the basis of the current results, it seems like the boxer are predisposed to producing $\operatorname{IgE}$ antibodies to outdoor allergens $(\mathrm{O}+)$, while the Rottweiler are predisposed to developing IgE hypersensitivity towards indoor allergens (I+). This is an interesting finding, as breed differences in allergen-specific IgE reactivity previously have been poorly studied in the dog; most studies on the subject have evaluated breed differences in total serum IgE levels. Also, several studies exist on breed predispositions to the disease CAD. Both total IgE levels and the occurrence of CAD have been documented to vary with breed $[7,10,11,17,18]$, and the results from the present study indicate that there also is breed variations in terms of which allergens dogs react to.

\section{Conclusions}

IgE hypersensitivity among Norwegian dogs with suspected CAD was most often associated with indoor allergens.
Outdoor allergens were of minor importance and IgE reactivity to flea saliva was rare. Serum levels of allergenspecific IgE varied with sex, age, geographical localisation, and sampling season. Breed differences in allergen-specific IgE levels were documented. Relative to the other breeds represented in the study sample, the boxer had a higher prevalence of elevated IgE levels to outdoor allergens, while the Rottweiler had a higher prevalence of elevated IgE levels to indoor allergens.

\section{Competing interests}

The results from the allergy tests were obtained from Dr. Baddaky AS, but the study was financed, monitored and conducted solely by the Norwegian School of Veterinary Science.

The authors have no financial or personal relationship with people or organisations that could inappropriately influence or bias the content of this paper.

\section{Authors' contributions}

$A A B$ and FLD initiated the study and collected data, performed the analysis as well as interpreted and presented the results. This was done as their final year research project at the Norwegian School of Veterinary Science. AAB drafted the manuscript. FLD prepared the figures and tables. AN helped with planning and conduction of data-analysis and with writing of the manuscript. BKS helped formulate research questions, aided in the planning of the study and interpretation of results as well as providing input to the manuscript. All authors have read and approved the final version of the manuscript.

\section{Acknowledgements}

The authors wish to thank Babette B. Taugbøl from Dr. Baddaky AS for making the database of test results available to us. We would also like to thank Dagfinn Nordhaug from ProfVet AS for his technical assistance regarding database administration.

\section{Author details}

${ }^{1}$ Department of Companion Animal Clinical Sciences, Faculty of Veterinary Medicine and Biosciences, Norwegian University of Life Sciences, PO box 8146 Dep., NO-0033, Oslo, Norway. ${ }^{2}$ Department of Production Animal Clinical Sciences, Faculty of Veterinary Medicine and Biosciences, Norwegian University of Life Sciences, PO box 8146 Dep., NO-0033, Oslo, Norway. ${ }^{3}$ Department of Basic Sciences and Aquatic Medicine, Faculty of Veterinary Medicine and Biosciences, Norwegian University of Life Sciences, PO box 8146 Dep., NO-0033, Oslo, Norway.

Received: 11 August 2014 Accepted: 22 November 2014

Published online: 05 December 2014

\section{References}

1. Halliwell R: Revised nomenclature for veterinary allergy. Vet Immunol Immunopathol 2006, 114:207-208.

2. Hill PB, DeBoer DJ: The ACVD task force on canine atopic dermatitis (IV): environmental allergens. Vet Immunol Immunopathol 2001, 81:169-186.

3. Halliwell RE, DeBoer DJ: The ACVD task force on canine atopic dermatitis (III): the role of antibodies in canine atopic dermatitis. Vet Immunol Immunopathol 2001, 81:159-167.

4. Marsella R, Sousa CA, Gonzales AJ, Fadok VA: Current understanding of the pathophysiologic mechanisms of canine atopic dermatitis. J Am Vet Med Assoc 2012, 241:194-207.

5. Olivry T, Hill PB: The ACVD task force on canine atopic dermatitis (VIII): is the epidermal lipid barrier defective? Vet Immunol Immunopathol 2001, 81:215-218

6. Theerawatanasirikul S, Sailasuta A, Thanawongnuwech R, Suriyaphol S: Alterations of keratins, involucrin and filaggrin gene expression in canine atopic dermatitis. Res Vet Sci 2012, 93:1287-1292.

7. Nødtvedt A, Egenvall A, Bergvall K, Hedhammar Å: Incidence of and risk factors for atopic dermatitis in a Swedish population of insured dogs. Vet Rec 2006, 159:241-246. 
8. Nødtvedt A, Guitian J, Egenvall A, Emanuelson U, Pfeiffer DU: The spatial distribution of atopic dermatitis cases in a population of insured Swedish dogs. Prev Vet Med 2007, 78:210-222

9. Hillier A, Griffin CE: The ACVD task force on canine atopic dermatitis (I): incidence and prevalence. Vet Immunol Immunopathol 2001, 81:147-151.

10. Griffin CE, DeBoer DJ: The ACVD task force on canine atopic dermatitis (XIV): clinical manifestations of canine atopic dermatitis. Vet Immunol Immunopathol 2001, 81:255-269.

11. Sousa CA, Marsella R: The ACVD task force on canine atopic dermatitis (II): genetic factors. Vet Immunol Immunopathol 2001, 81:153-157.

12. Olivry T, DeBoer DJ, Favrot C, Jackson HA, Mueller RS, Nuttall T, Prélaud P: Treatment of canine atopic dermatitis: 2010 clinical practice guidelines from the International Task Force on Canine Atopic Dermatitis. Vet Dermatol 2010, 21:233-248.

13. Favrot C, Steffan J, Seewald W, Picco F: A prospective study on the clinical features of chronic canine atopic dermatitis and its diagnosis. Vet Dermatol 2010, 21:23-31.

14. DeBoer DJ, Hillier A: The ACVD task force on canine atopic dermatitis $(\mathrm{XVI})$ : laboratory evaluation of dogs with atopic dermatitis with serum-based "allergy" tests. Vet Immunol Immunopathol 2001, 81:277-287.

15. Meury S, Molitor V, Doherr MG, Roosje P, Leeb T, Hobi S, Wilhelm S, Favrot C: Role of the environment in the development of canine atopic dermatitis in Labrador and golden retrievers. Vet Dermatol 2011, 22:327-334.

16. Nødtvedt A, Bergvall K, Sallander M, Egenvall A, Emanuelson U, Hedhammar $\AA$ : A case-control study of risk factors for canine atopic dermatitis among boxer, bullterrier and West Highland white terrier dogs in Sweden. Vet Dermatol 2007, 18:309-315.

17. Lauber B, Molitor V, Meury S, Doherr MG, Favrot C, Tengvall K, Bergvall K, Leeb T, Roosje P, Marti E: Total IgE and allergen-specific IgE and IgG antibody levels in sera of atopic dermatitis affected and non-affected Labrador- and Golden retrievers. Vet Immunol Immunopathol 2012, 149:112-118.

18. Griot-Wenk ME, Busato A, Welle M, Racine BP, Weilenmann R, Tschudi P, Tipold A: Total serum $\lg E$ and $\lg A$ antibody levels in healthy dogs of different breeds and exposed to different environments. Res Vet Sci 1999, 67:239-243.

19. Racine BP, Marti E, Busato A, Weilenmann R, Lazary S, Griot-Wenk ME: Influence of sex and age on serum total immunoglobulin $E$ concentration in Beagles. Am J Vet Res 1999, 60:93-97.

20. Stedman K, Lee K, Hunter S, Rivoire B, McCall C, Wassom D: Measurement of canine $\lg E$ using the alpha chain of the human high affinity $\lg E$ receptor. Vet Immunol Immunopathol 2001, 78:349-355.

21. ALLERCEPT Serum IgE testing [http://www.heska.com/Products/ALLERCEPT/ ALLERCEPT-Testing.aspx]

22. Allergy Testing in the Dog \& Cat [http://axiomvetlab.com/Allergy\% 20brochure.pdf]

23. Postnummerregister [http://www.bring.no/hele-bring/produkter-og-tjenester/ brev-og-postreklame/andre-tjenester/postnummertabeller]

24. Regionale inndelinger: En Oversikt over Standarder i Norsk Offisiell Statistikk [http://www.ssb.no/emner/00/00/20/nos_c513/]

25. Estimate Confidence Limits for a Mean [http://epitools.ausvet.com.au/content. php?page=CIMean]

26. Chi-squared test for $r \times$ c Contigency Table [http://epitools.ausvet.com.au/ content.php?page=chi_sq]

27. Allergiprogram Hund og Katt [http://www.drbaddaky.no/products/ allergiprogram_hund_katt]

28. Saridomichelakis MN, Marsella R, Lee KW, Esch RE, Farmaki R, Koutinas AF: Assessment of cross-reactivity among five species of house dust and storage mites. Vet Dermatol 2008, 19:67-76.

29. Vollset I: Atopic dermatitis in Norwegian dogs. Nord Vet Med 1985, 37:97-106.

30. Bergvall KE, Saevik BK, Saijonmaa-Koulumies L, Holm B, Holm L, Hedhammar Å, Kristensen F: Demographics and clinical picture of nonseasonal canine atopic dermatitis - observations in 63 dogs. Vet Dermatol 2002, 13:211-229.

31. Sousa CA, Halliwell RE: The ACVD task force on canine atopic dermatitis (XI): the relationship between arthropod hypersensitivity and atopic dermatitis in the dog. Vet Immunol Immunopathol 2001, 81:233-237.

32. Kart Med Nedbørnormal for Norge [http://met.no/Klima/Klimastatistikk/ Klimanormaler/Nedbor/]
33. Arlian LG, Neal JS, Vyszenski-Moher DL: Reducing relative humidity to control the house dust mite Dermatophagoides farinae. J Allergy Clin Immunol 1999, 104:852-856.

34. Registrering Av Pollen Og Sporer 2012 [http://www.pollenvarslingen.no/Files/ Årsrapport2012_NY_komprimmert.pdf]

35. Korsgaard J: Epidemiology of house-dust mites. Allergy 1998, 53(Suppl 48):36-40

doi:10.1186/s13028-014-0081-z

Cite this article as: Bjelland et al.: Prevalence of and risk factors for increased serum levels of allergen-specific $\lg \mathrm{E}$ in a population of Norwegian dogs. Acta Veterinaria Scandinavica 2014 56:81.

\section{Submit your next manuscript to BioMed Central and take full advantage of:}

- Convenient online submission

- Thorough peer review

- No space constraints or color figure charges

- Immediate publication on acceptance

- Inclusion in PubMed, CAS, Scopus and Google Scholar

- Research which is freely available for redistribution

Submit your manuscript at www.biomedcentral.com/submit
C) BioMed Central 